\title{
RIESZ OPERATORS AND FREDHOLM PERTURBATIONS
}

\author{
BY MARTIN SCHECHTER
}

\author{
Communicated by B. Yood, June 21, 1968
}

1. Introduction. Let $X$ be a Banach space, and let $B(X)$ denote the space of bounded linear operators on $X$. An operator $A \in B(X)$ is called a Fredholm operator if

1. $\alpha(A)$, the dimension of the null space $N(A)$ of $A$, is finite;

2. the range $R(A)$ of $A$ is closed in $X$;

3. $\beta(A)$ the codimension of $R(A)$, is finite.

The set of Fredholm operators on $X$ is denoted by $\Phi(X)$. An operator $E \in B(X)$ is called a Riesz operator if $E-\lambda \in \Phi(X)$ for all scalars $\lambda \neq 0$. For further discussion of such operators we refer to $[1$, p. 323], [2], [3], [4], [5], [9].

An operator $E \in B(X)$ is called a Fredholm perturbation if $A+E$ $\in \Phi(X)$ for all $A \in \Phi(X)$. In this paper we investigate the connection between Riesz operators and Fredholm perturbations. Our work complements the results of [2], [3] and [6].

2. Riesz operators. Let $R(X)$ denote the set of Riesz operators on $X$.

Lemma 1. $E \in R(X)$ if and only if $I+\lambda E \in \Phi(X)$ for all scalars $\lambda$.

Proof. If $E \in R(X)$, the statement is true for $\lambda=0$. Otherwise $E+I / \lambda \in \Phi(X)$. Hence $I+\lambda E \in \Phi(X)$. Conversely, if $\mu \neq 0$, then $\mu(I+E / \mu) \in \Phi(X)$ showing that $E+\mu \in \Phi(X)$.

The set $K(X)$ of compact operators on $X$ is a closed, two-sided ideal in $B(X)$. Let $\pi$ be the natural quotient map of $B(X)$ into $B(X) / K(X)$.

LEMma 2 [7]. $A \in \Phi(X)$ if and only if $\pi(A)$ is invertible in $B(X) / K(X)$.

Lemma 3 [9], [1]. $E \in R(X) \Leftrightarrow\left\|\pi(E)^{n}\right\| \|^{1 / n} \rightarrow 0$ as $\mathrm{n} \rightarrow \infty$.

For any two operators $A, B \in B(X)$ we shall write $A \bigcup_{x} B$ when $A B-B A$ is a compact operator on $X$. The reason for the notation is that $\pi(A B)=\pi(B A)$ in this case. Such operators are said to "almost commute."

Lemma 4. If $E \in R(X)$ and $K \in K(X)$, then $E+K \in R(X)$.

Proof. $\pi(E+K-\lambda)=\pi(E-\lambda)$. 
Lemma 5. If $E \in R(X), B \in B(X)$ and $B \bigcup_{x} E$, then $E B$ and $B E$ are in $R(X)$.

PROOF. $\left\|\pi(E B)^{n}\right\|^{1 / n}=\left\|\pi(B)^{n} \pi(E)^{n}\right\|^{1 / n}$.

$$
\leqq\|\pi(B)\|\left\|\pi(E)^{n}\right\| 1 / n \rightarrow 0 \text { as } n \rightarrow \infty .
$$

Lemma 6 [8]. If $A \in \Phi(X)$, then there is a $\hat{A} \in \Phi(X)$, such that

$$
\pi(\hat{A} A)=\pi(A \hat{A})=\pi(I) .
$$

Lemma 7. If $E \in R(X), A \in \Phi(X)$ and $A \bigcup_{\pi} E$, then $\hat{A}+E \in \Phi(X)$.

Proof. $\pi[A(E+\hat{A})]=\pi[(E+\hat{A}) A]=\pi(E A+I)$. Since $E A \in R(X)$ (Lemma 5), $E A+I$ is $\Phi(X)$ and $\pi(E A+I)$ is invertible in $B(X) / K(X)$. Hence the same is true of $\pi(E+\hat{A})$, showing that $E+\hat{A} \in \Phi(X)$.

Lemma 8. If $A \in \Phi(X), E \in R(X)$, and $A \bigcup_{\pi} E$, then $\hat{A} \bigcup_{\pi} E$.

Proof. $\pi(\hat{A} E)=\pi(\hat{A} E A \hat{A})=\pi(\hat{A} A E \hat{A})=\pi(E \hat{A})$.

Theorem 9. If $A \in \Phi(X), E \in R(X)$ and $A \bigcup_{\pi} E$, then $A+E \in \Phi(X)$.

Proof. $\hat{A} \in \Phi(X)$ and $\hat{A} U_{\pi} E$ (Lemmas 6 and 8). Thus $A+E \in \Phi(X)$ (Lemma 7).

Lemma 10. Suppose $A \in \Phi(X)$ and $E \in B(X)$. Then $\lambda E+A \in \Phi(X)$ for all $\lambda$ if and only if $E \hat{A} \in R(X)$.

Proof. If $\lambda E+A \in \Phi$, then $\pi[(\lambda E+A) \hat{A}]=\pi[\hat{A}(\lambda E+A)]$ $=\pi[\lambda E \hat{A}+I]$ is invertible in $B(X) / K(X)$. Hence $E \hat{A} \in R(X)$. Conversely, if $E \hat{A} \in R(X)$, then $\pi(\lambda E \hat{A}+I)$ is invertible for each $\lambda$. Hence so is $\pi(\lambda E+A)$.

Lemma 11. Suppose $A \in \Phi(X)$ and $E \in B(X)$. Then $E A \in R(X)$ if and only if $A E \in R(X)$.

Proof. If $E A \in R(X)$, then $\lambda E A+I \in \Phi(X)$ for all $\lambda$. Hence so is $\lambda E+\hat{A}$ and consequently so is $\lambda A E+I$. Therefore $A E \in R(X)$.

THEOREM 12. The operator $E \in B(X)$ is in $R(X)$ if and only if $A+E$ $\in \Phi(X)$ for all $A \in \Phi(X)$ such that $A \bigcup_{\pi} E$.

Proof. By Theorem 9 we need only show the "if" part. To do this we merely take $A=\lambda \neq 0$.

Theorem 13. If $E_{1}, E_{2} \in R(X)$ and $E_{1} \bigcup_{\pi} E_{2}$, then $E_{1}+E_{2} \in R(X)$.

Proof. If $\lambda \neq 0, \lambda+E_{1} \in \Phi(X)$. By Theorem 9 so is $\lambda+E_{1}+E_{2}$. Thus $E_{1}+E_{2} \in R(X)$. 
3. Fredholm perturbations. Let $F(X)$ denote the set of those $E \in B(X)$ such that $A E \in R(X)$ for all $A \in \Phi(X)$. We now characterize this set.

Lemma 14. $E \in F(X)$ if and only if $I+A E \in \Phi(X)$ for all $A \in \Phi(X)$.

Proof. Use Lemma 1.

Theorem 15. $E \in F(X)$ if and only if $A+E \in \Phi(X)$ for all $A \in \Phi(X)$. Thus $F(X)$ coincides with the set of Fredholm perturbations.

Proof. If $E \in F(X)$ and $A \in \Phi(X)$, then $\hat{A} E \in R(X)$ (Lemma 6). Thus $I+\hat{A} E \in \Phi(X)$ (Lemma 1). Thus $A(I+\hat{A} E) \in \Phi(X)$ showing that $\pi(A+E)$ is invertible. Hence $A+E \in \Phi(X)$. Conversely, suppose $A+E \in \Phi(X)$ for all $A \in \Phi(X)$. Let $A$ be a particular operator in $\Phi(X)$. Then $\lambda \hat{A}+E \in \Phi(X)$ for all $\lambda \neq 0$. Hence the same is true for $A(\lambda \hat{A}+E)$. This shows that $\pi(\lambda+A E)$ is invertible for each $\lambda \neq 0$. Hence $A E$ $\in R(X)$. Since this is true for all $A \in \Phi(X)$, we have $E \in F(X)$.

Corollary 16. If $E_{1}, E_{2} \in F(X)$, then $E_{1}+E_{2} \in F(X)$.

LEMma 17. For each $B \in B(X)$, there are operators $A_{1}, A_{2}$ in $\Phi(X)$ such that $B=A_{1}+A_{2}$.

Proof. For $\lambda$ sufficiently large, $A_{1}=\lambda I+B$ is in $\Phi(X)$ (cf., e.g., [4], [8]). Take $A_{2}=-\lambda I$.

Corollary 18. If $E \in F(X)$, then $B E \in F(X)$ for all $B \in B(X)$.

Proof. By Lemma $17, B=A_{1}+A_{2}$, where $A_{j} \in \Phi(X)$. If $A$ is any operator in $\Phi(X)$, then $A A_{j} E \in R(X)$. Thus $A_{j} E \in F(X)$. Hence $B E=A_{1} E+A_{2} E \in F(X)$ (Corollary 16).

Corollary 19. If $E \in F(X)$, then $E A \in R(X)$ for all $A \in \Phi(X)$.

Proof. Lemma 11.

Corollary 20. If $E \in F(X)$, then $E B \in F(X)$ for all $B \in B(X)$.

Proof. See the proof of Corollary 18.

Corollary 21. If $E_{n} \in F(X)$ and $E_{n} \rightarrow E$ in $B(X)$, then $E \in F(X)$.

Proof. If $A \in \Phi(X)$, we can take $n$ so large that $A-\left(E_{n}-E\right) \in \Phi(X)$ (cf., e.g., [4]). Hence $A-\left(E_{n}-E\right)+E_{n} \in \Phi(X)$ (Theorem 15). This shows that $E \in F(X)$.

COROLlary 22. $F(X)$ is a closed two-sided ideal.

Proof. Corollaries 18, 20, 21. 
4. Semi-Fredholm operators. Let $\Phi_{+}(X)$ denote the set of operators $A \in B(X)$ such that $\alpha(A)<\infty$ and $R(A)$ is closed in $X$. Clearly $\Phi_{+}(X)$ contains $\Phi(X)$.

THEOREM 23. $A$ is in $\Phi_{+}(X)$ if and only if $\alpha(A-K)<\infty$ for all $K \in K(X)$.

Proof. If $A \in \Phi_{+}(X)$ and $K \in K(X)$, then $A-K \in \Phi_{+}(X)$ (cf. [8], [4]). In particular, $\alpha(A-K)<\infty$. Conversely, suppose $A$ is not in $\Phi_{+}(X)$. Then there are sequences $\left\{x_{k}\right\} \subseteq X,\left\{x_{k}^{\prime}\right\} \subseteq X^{\prime}$ such that $\left\|x_{k}\right\|=1,\left\|x_{k}^{\prime}\right\| \leqq 2^{k-1}, x_{j}^{\prime}\left(x_{k}\right)=\delta_{j k},\left\|A x_{k}\right\| \leqq 2^{1-2 k}$ (cf. [6]). Set

$$
K_{n} x=\sum_{1}^{n} x_{k}^{\prime}(x) A x_{k}, \quad n=1,2, \cdots
$$

Then for $n>m$

$$
\left\|\left(K_{n}-K_{m}\right) x\right\| \leqq \sum_{m+1}^{n} 2^{k-1} 2^{1-2 k}\|x\|,
$$

showing that $\left\|K_{n}-K_{m}\right\| \rightarrow 0$ as $m, n \rightarrow \infty$. Thus $K_{n} \rightarrow K$, where

$$
K x=\sum_{1}^{\infty} x_{k}^{\prime}(x) A x_{k} .
$$

Now $K x=A x$ for $x$ equal to any of the $x_{k}$ and hence also for any linear combination. Since the $x_{k}$ are linearly independent, it follows that $\alpha(A-K)=\infty$. This completes the proof.

Corollary 24. $A \in \Phi(X)$ if and only if $\alpha(A-K)<\infty$ and $\beta(A-K)$ $<\infty$ for all $K \in K(X)$.

Proof. The "only if" part is well known (cf., e.g., [4]). To prove the "if" part, note that Theorem 23 implies that $A \in \Phi_{+}(X)$. Since $0 \in K(X), \beta(A)=\beta(A-0)<\infty$. Thus $A \in \Phi(X)$.

Let $F_{+}(X)$ denote the set of all $E \in B(X)$ such that $A+E \in \Phi_{+}(X)$ for all $A \in \Phi_{+}(X)$.

Corollary 25. If $E_{1}, E_{2} \in F_{+}(X)$, then $E_{1}+E_{2} \in F_{+}(X)$.

Theorem 26. $E \in F_{+}(X)$ if and only if $\alpha(A-E)<\infty$ for all $A \in \Phi_{+}(X)$.

Proof. If $E \in F_{+}(X)$ and $A \in \Phi_{+}(X)$, then $A-E \in \Phi_{+}(X)$ by definition. Hence $\alpha(A-E)<\infty$. If $A \in \Phi_{+}(X)$ and $A-E$ is not in $\Phi_{+}(X)$, then there is a $K \in K(X)$ such that $\alpha(A-E-K)=\infty$ (Theorem 23). Set $C=A-K$. Then $C \in \Phi_{+}(X)$ and $\alpha(C-E)=\infty$. This proves the theorem. 
Theorem 27. $E \in F(X)$ if and only if $\alpha(A-E)<\infty$ for all $A \in \Phi(X)$.

Proof. If $E \in F(X)$ and $A \in \Phi(X)$, then $A-E \in \Phi(X)$ (Theorem 15). Thus $\alpha(A-E)<\infty$. Conversely, suppose $\alpha(A-E)<\infty$ for all $A \in \Phi(X)$. Let $A$ be any particular operator in $\Phi(X)$. Then $(A-K) / \lambda \in \Phi(X)$ for each $K \in K(X)$ and $\lambda \neq 0$. Hence $\alpha(A-\lambda E-K)$ $<\infty$ for all $\lambda$ and all $K \in K(X)$. By Theorem 23, $A-\lambda E \in \Phi_{+}(X)$ for each $\lambda$. In particular, this is true for $0 \leqq \lambda \leqq 1$. Now if $\beta(A-E)$ were infinite, it would follow that $\beta(A)=\infty[4$, Theorem 7.1]. But this is contrary to assumption. Hence $A-E \in \Phi(X)$. Since this is true for any $A \in \Phi(X)$, the proof is complete.

Corollary 28. $F_{+}(X) \subseteq F(X)$.

Lemma 29. If $E_{n} \in F_{+}(X)$ and $E_{n} \rightarrow E$, then $E \in F_{+}(X)$.

Proof. See Corollary 21.

LEMma 30. If $E \in F_{+}(X)$, then $A E$ and $E A$ are in $F_{+}(X)$ for all $A \in \Phi(X)$.

Proof. If $A \in \Phi(X)$ and $C \in \Phi_{+}(X)$, then $E+\hat{A} C \in \Phi_{+}(X)$. Thus $A(E+\hat{A} C)$ and consequently $A E+C$ are also in $\Phi_{+}(X)$. This means that $A E \in F_{+}(X)$. A similar argument holds for $E A$.

LEMmA 31. If $E \in F_{+}(X)$, then $B E$ and $E B$ are in $F_{+}(X)$ for all $B \in B(X)$.

Proof. See Corollary 18.

Corollary 32. $F_{+}(X)$ is a closed, two-sided ideal.

Proof. Lemmas 29 and 31.

5. Remarks. $R(X)$ is not an ideal [3]. We see from Corollary 22 that $F(X)$ is the largest ideal contained in $R(X)$. Moreover, operators in $R(X)$ are characterized by the fact that each of them behaves like a Fredholm perturbation with respect to Fredholm operators which almost commute with it (Theorem 12).

Theorem 23 says that an operator in $\Phi_{+}(X)$ cannot coincide with a compact operator on any infinite dimensional subspace, and that this property characterizes these operators. Theorem 26 says that an operator is in $F_{+}(X)$ if and only if it does not coincide with a $\Phi_{+}(X)$ operator on any infinite dimensional subspace. Theorem 27 makes a similar statement for $F(X)$.

\section{BIBLIOGRAPHY}

1. Jean Dieudonne, Foundations of modern analysis, Academic Press, New York, 1960. 
2. David Kleinecke, Almost-finite, compact and inessential operators, Proc. Amer. Math. Soc. 14 (1963), 863-868.

3. S. R. Caradus, Operator of Riesz type, Pacific J. Math. 18 (1966), 61-71.

4. I. C. Gohberg and M. G. Krein, Fundamental aspects of defect numbers, root numbers, and indices of linear operators, Uspehi Mat. Nauk. 12 (1957), 43-118.

5. Shmuel Kaniel and Martin Schechter, Spectral theory for Fredholm operators, Comm. Pure Appl. Math. 16 (1963), 423-448.

6. I. A. Feldman, I. C. Gohberg and A. S. Markus, Normally solvable operators and ideals associated with them, Izv. Moldavsk Filiala Akad. Nauk. SSSR, 10 (1960), 51-69.

7. Bertram Yood, Difference algebras of linear transformations on a Banach space, Pacific J. Math. 4 (1954), 615-636.

8. - Properties of linear transformations preserved under addition of a completely continuous transformation, Duke Math. J. 18 (1951), 599-612.

9. A. F. Ruston, Operators with Fredholm theory, J. London Math. Soc. 29 (1954), 318-326.

Belfer Graduate School of Science, Yeshiva University

\title{
THE CENTRALIZER OF A REGULAR UNIPOTENT ELEMENT IN A SEMISIMPLE ALGEBRAIC GROUP
}

\author{
BY BETTY LOU 1
}

Communicated by Louis Auslander, June 14, 1968

The following question was posed by Springer [2]: is the centralizer $G_{x}$ of a regular unipotent element $x$ in a semisimple algebraic group $G$ abelian? In this paper we shall give an affirmative answer and also find the number of disjoint components of $G_{x}$ if it is reducible. The problem is easily reduced to the case in which $G$ is simple, which we henceforth assume. As proved by Springer in [2], reducibility occurs only when the type of $G$ and the characteristic $p$ of the base field $\Phi$ are related as follows: $C_{n}(n \geqq 2)$ and $D_{n}(n \geqq 4)$ with $p=2$ (here $B_{n}$ is a homomorphic image of $C_{n}$ and need not be considered); $F_{4}, G_{2}$, $E_{6}, E_{7}$, with $p=2,3$ and $E_{8}$ with $p=2,3,5$.

We shall now sketch our development. We recall that an element $x$ of $G$ is regular if its centralizer $G_{x}$ has dimension equal to the rank, say $r$, of $G$, and that an element is unipotent if its eigenvalues are all 1. Relative to a Cartan decomposition of $G$ let $U$ be the maximal

1 The results are part of the author's Ph.D. thesis at the University of California at Los Angeles. The author wishes to thank Professor Robert Steinberg for his patient guidance during the preparation of this work. 